\title{
Research Paper \\ The Comparison of Executive Functions, Risk Behaviors, and Academic Motivation Among Adolescents With Chronology Type Morningness and Eveningness
}

\author{
Zahra Lariche ${ }^{1}, *$ Sayed Abbas Haghayegh ${ }^{2}$
}

1. MA Student, Department of Clinical Psychology, Najafabad Branch, Islamic Azad University, Najafabad, Iran.

Received: 16 Oct. 2016 Accepted: 18 Feb. 2017

Key words: Chronology as topic, Executive functions, Risky behaviors, Academic motivation
Citation: Lariche Z, Haghayegh SA. [The Comparison of Executive Functions, Risk Behaviors, and Academic Motivation Among Adolescents With Chronology Type Morningness and Eveningness (Persian)]. Iranian Journal of Psychiatry and Clinical Psychology. 2018; 23(4):438-453. https://doi.org/10.29252/NIRP.IJPCP.23.4.438

https://doi.org/10.29252/NIRP.IJPCP.23.4.438

$\begin{array}{ll}\text { Extended Abstract } & \begin{array}{l}\text { The psychological status of adolescents is not stable, and } \\ \text { most of the behavioral problems occur at this stage [9]. The } \\ \text { executive functions are neurological structures that are im- } \\ \text { 1. Introduction }\end{array} \\ \begin{array}{l}\text { portant to psychological functions such as consciousness } \\ \text { and thinking [13]. It has been observed that changes in the } \\ \text { ences in bio-rhythms that induced to differ- } \\ \text { ences in bio and psychological functions } \\ \text { chronotype of adolescents lead to lack of coordination and } \\ \text { il]. Bio-rhythms can be divided into morn- } \\ \text { functions in some of the activities related to academic per- } \\ \text { formance [18]. In comparison with other age groups, ado- } \\ \text { lescents are more prone to risky behaviors. Manifestations }\end{array}\end{array}$

Objectives This research was conducted with the goal of comparison of executive functions, risk behaviors, and academic motivation among adolescents with chronology type morningness and eveningness. Methods This is a comparative study in a population of high school students in Isfahan in 2014-15 academic year. Accessible sampling was used as a sampling method in two phases. In the first phase 320 students were selected, and in the second phase, 106 students with different chronology types were chosen as a final sample. The data gathered by using Horne and Östberg's Morningness-eveningness questionnaire, Mohammadi and his colleagues' Iranian Adolescents Risk Scale, Abdekhodaee's Achievement motivation questionnaire, and Wisconsin card sorting test. Independent samples $t$ test and MannWhitney $\mathrm{U}$ test were employed for analyzing data using SPSS-21.

Results Significant relationship was found between two aspects of executive functions containing preservation error and incorrect answers responses, and chronology types $(P<0.05)$, whereas no significant relationship existed in other aspects. In addition, morningness and eveningness chronology types were significantly different among adolescents in terms of risky behaviors and academic motivation $(P<0.05)$. Conclusion The adolescents with morningness chronology type performed better in executive function and academic motivation in comparison with eveningness type and had lower risky behaviors.

* Corresponding Author:

Sayed Abbas Haghayegh, PhD

Address: Department of Psychology, Najafabad Branch, Islamic Azad University, Najafabad, Iran.

Tel: +98 (31) 42293030

E-mail: abbas_haghayegh@yahoo.com 
of these behaviors increase in middle adolescence stage and decrease in late adolescence stage [19].

One of the factors of academic problems is academic motivation [28]. Motivation is a common concept of educational systems [29]. The study of high-risk behaviors in terms of the consequences for life, health, psychological and social development of adolescents such as mental and psychological disorders, including depression, illness, and even death [20], AIDS and sexual diseases [21], dropout, school escape, academic and occupation failure [22], and involvement in various offenses is of great significance. Drug use, violence, and sexual behaviors are responsible for many deaths in adolescence and early adulthood [23]. This study was undertaken with the goal to compare executive functions, risk behaviors, and academic motivation among adolescents with morningness and eveningness chronology types.

\section{Method}

The research plan was a comparative study and population was high school students in Isfahan in the academic year 2014-15. Accessible sampling was used as a sampling method in two phases. In the first phase, 320 students were selected from the population. Sample selection was done in coordination with the Department of Education, Isfahan. Then, one of the education districts from Isfahan was selected. After identifying the schools, the date and time of attending schools were decided in consultation with the school administrators. We collected the data using Horne and Östberg's Morningness-Eveningness Questionnaire (1976), Mohammadi et al. Scale of Iranian Adolescents Risk, Abdekhodaee's Achievement Motivation Questionnaire, and Wisconsin Card Sorting Test.

The students whose scores were in the range of 41-58 in Morningness-Eveningness Questionnaire were eliminated from the study. In the final sample,106 students were included in the morningness chronotype (scores in the range of 59-86) and eveningness chronotypes (scores in the range of 16-41). The participants were fully aware of the study's objectives and participated on their own will. The participants were allowed to leave the project at any time. Inclusion criterion to participate in the test were being high-school students, and exclusion criteria consisted of failure to answer the questions and diagnosis of epilepsy. Data obtained from the study firstly were analyzed using SPSS 21 software by descriptive statistics, inferential analysis (after study of normality with KolmogorovSmirnov test), and equality of variances (with Levene test) presumptions) including independent-samples t test and Mann-Whitney U test.

\section{Results}

We observed no significant differences in demographics variables between the two groups, so we did not need to control them. Difference between the two aspects of executive functions and chronology types appeared significant $(\mathrm{P}<0.05)$. No discernible difference was found in other aspects. Further, among adolescents, significant difference was found between morningness and eveningness chronology types with regard to risky behaviors and academic motivation $(\mathrm{P}<0.05)$. No significant difference was observed in any other variables.

\section{Discussion}

This research was conducted with the goal of comparing executive functions, risk behaviors, and academic motivation among adolescents with morningness and eveningness chronology types. Obtained results are discussed in the following. The results obtained for time types and executive functions showed that there was a significant difference in the dimensions of executive functions (perseveration and inadequate responses) between the two groups. In fact, perseveration and inadequate responses are likely to be more at eveningness type adolescents. Executive functions may be compromised at school in the morning. The adolescents tested in this study at a desirable time performed better than those who were tested at an undesirable time.

Researchers have described different functions for sleep. When people are asleep, their brains are active; this activity is necessary for all body functions such as memory enhancement, learning, cognitive development, mental health, immune function, and physical growth and recovery. It is noteworthy that sleep plays an important role in people's daily activities. Given that sleep-awakening rhythms and activity-rest of the eveningness type are delayed in those with morningness type, eveningness type people sleep late and wake up late in the morning and compensate for their sleep deprivation. But for teenagers of the eveningness type, this lack of sleep is less compensated during the school.

The results for time types and high-risk behaviors indicated that there was a significant difference between adolescents of morningness and morningness in terms of high-risk behaviors and the dimensions of high-risk behaviors (e.g. drug, alcohol, cigarette, violence, sexual relations with the opposite sex, and dangerous driving). In fact, high-risk behaviors are higher in adolescents of the eveningness type. Types of morningness are likely to be futuristic, and as a result, they will look at the consequences of their behavior in the future; while the eveningness types tend to enjoy the present time and they 
are more eager to experience the instant pleasure [27]. It can be argued that compared with morningness people, eveningness ones are more prone to consume alcohol and smoke cigarettes to compensate for and eliminate drowsiness since they have more sleep, emotional, and behavioral problems during the week and weekends.

\section{Ethical Considerations}

\section{Funding}

This paper was extracted from the MA thesis of the first author in the Department of Psychology, Najafabad Branch, Islamic Azad University, Najafabad, Iran.

\section{Conflict of Interest}

The authors declare no conflict of interest.

\section{Acknowledgments}

We appreciate all participants in this study and those who helped us in conducting this research. 


\title{
مقايسه كاركردهاى اجرايى، رفتارهاى برخطر و انكَيزه تحصيلى بين نوجوانان با تيڤهاى زمانى

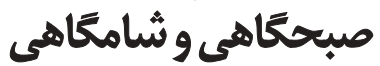

\author{
زهرا لاريجه'، "سيد عباس حقايق' \\ 1- دانشجوى كارشناسى ارشد، كروه روانشئاسى بالينى، واحد نجفآباد، دانشعاه آزاد اسلامى، نجفآباد، ايران.

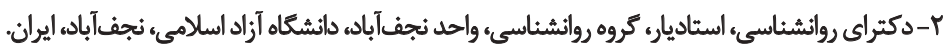

\begin{abstract}
حكبد

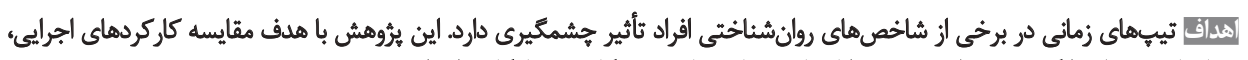

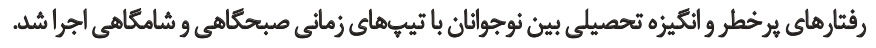

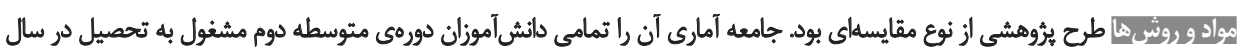

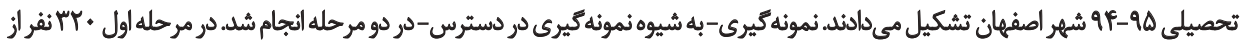

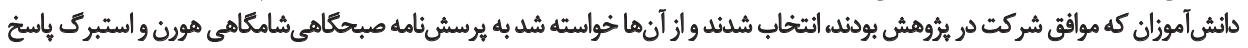

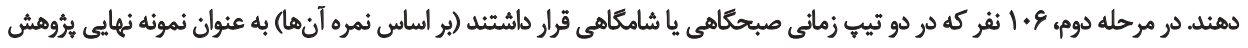

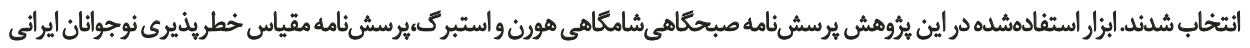

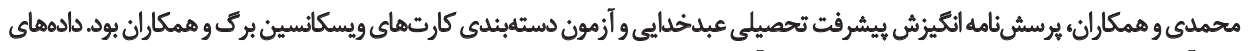

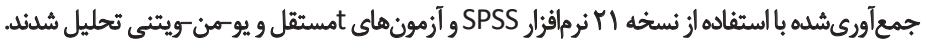

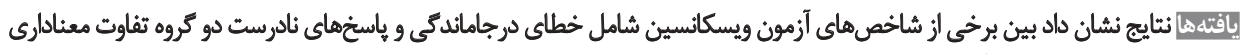

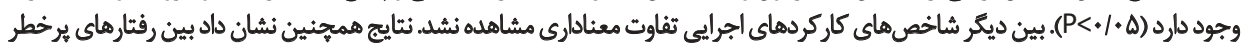

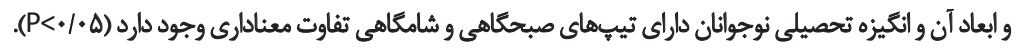

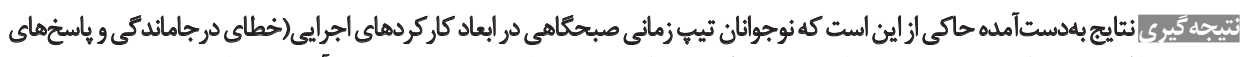

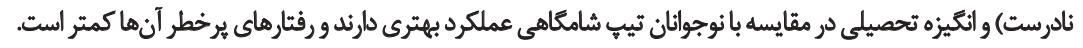

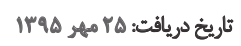

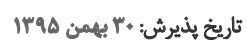

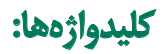 تيبهائ زمائي، كاركرونهاي

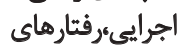 برخطر ائكيزه نحصيلي

روزانه، مقابله با استرس، بهره هوشى، نقايص شناختى، توجه

مقدمه

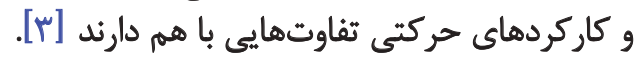

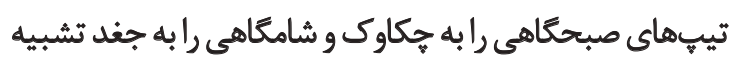

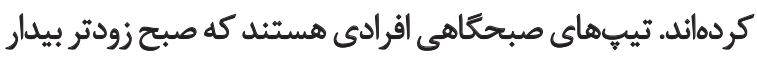

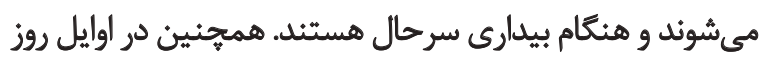

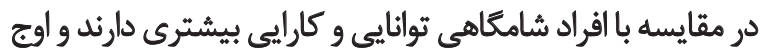

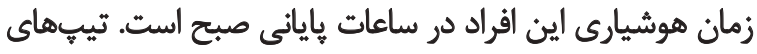

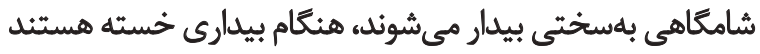

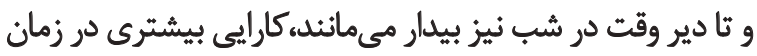

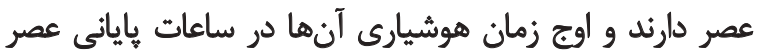

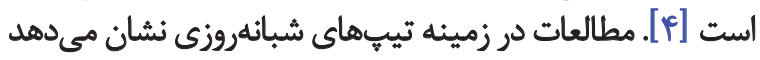

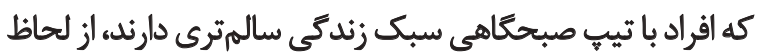

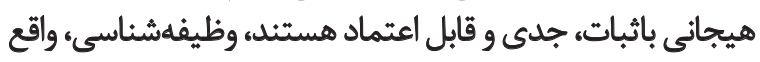

تيبهاى زمانى' به تفاوتهاى فردى در ريتمهاى زيستى

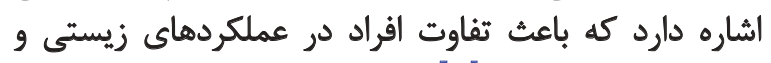

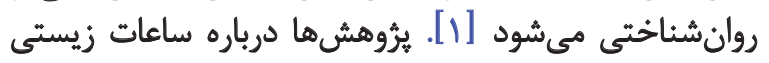

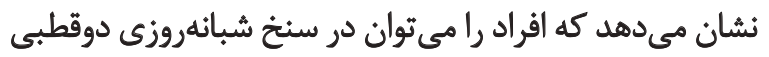

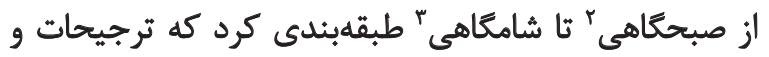

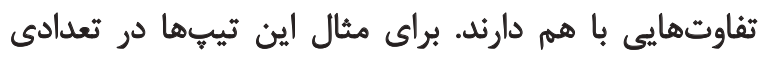

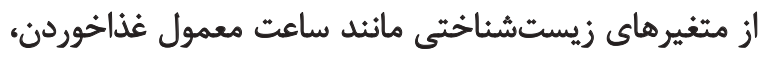

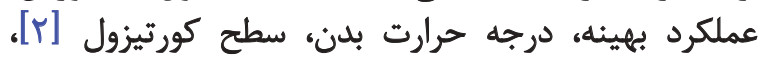
ترشح ملاتونين و متغيرهاى روانشينه دران بداختى مانئد عملكرد
1. Chronotypes
2. Morningness
3. Eveningness 
هيجان و رفتار و امكان دستيابى به اهداف روزمره تعريف كرد كه

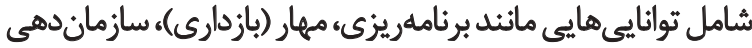

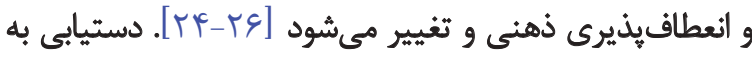

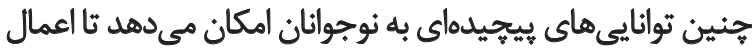

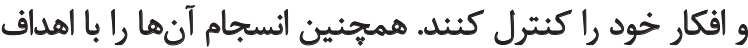

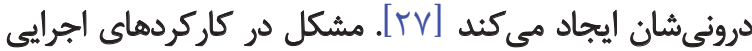

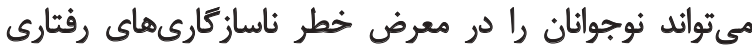

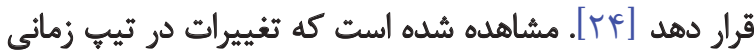

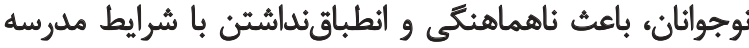

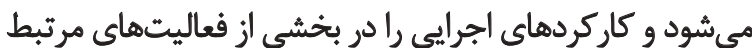

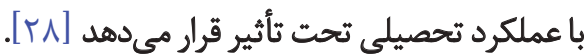
دوره نوجوانى كه باتغييرات جسمانى، روانشناختى واجتماعى خئي

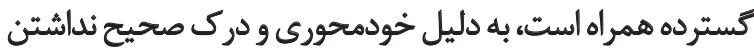

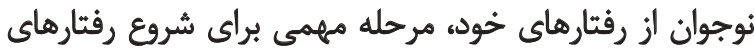

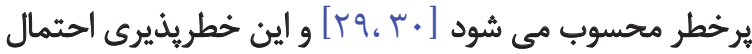

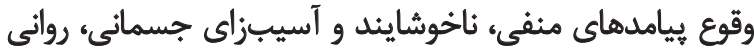

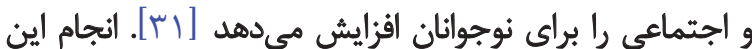

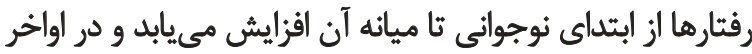

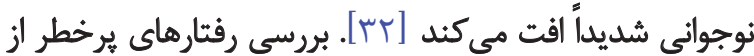

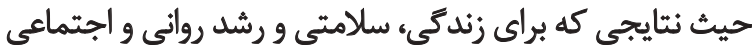

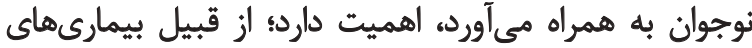
روحى و روانى مانيد افسردگى، ناخوشى و حتى مرى لـى [بس]، ايدز

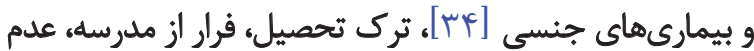

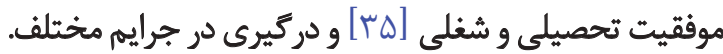
مصرف مواد مخدر، خشويت و رفتارهاى جنسى عامل بسيارى

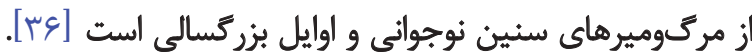

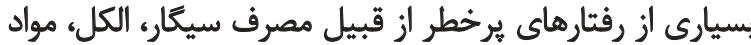

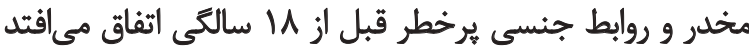

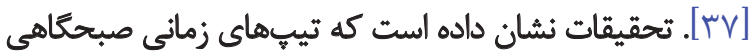

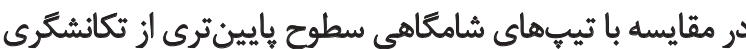

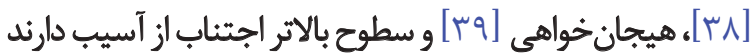

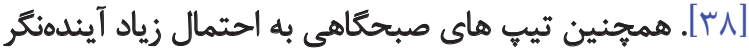

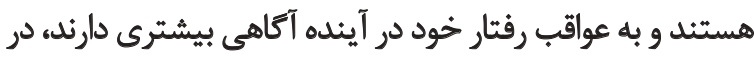
حالى كه تيب شامعاهى خودكنترلى كمترى دارند [ [ [ ]

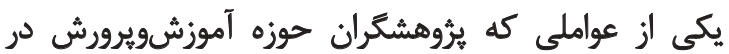

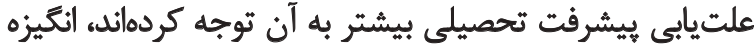

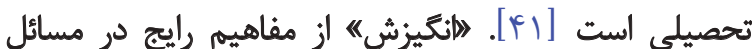

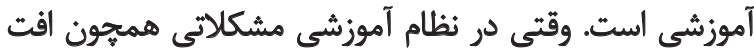

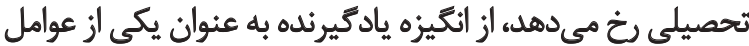

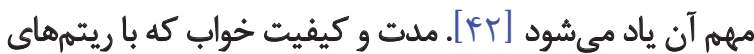
شبانهروزى مرتبط است، ممكن است بر موفقيت تحصيلى افراد
كرايي و اعتمادبهنفس در آنها بيشتر است و در زميئه كارى

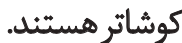

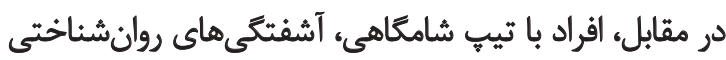

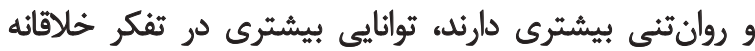

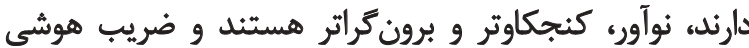

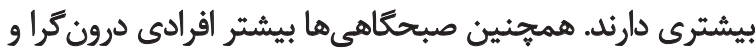
شامعاهى ها بيشتر برون

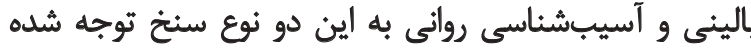

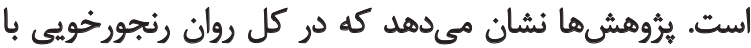

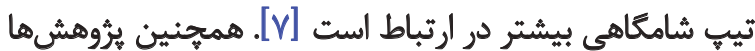

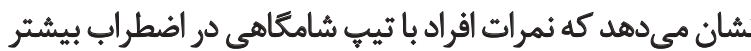

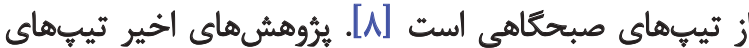

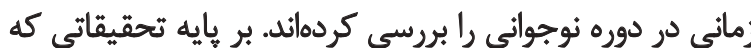

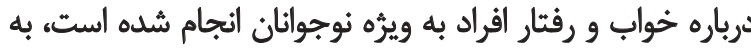

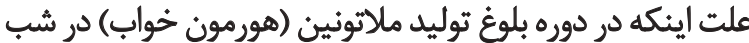

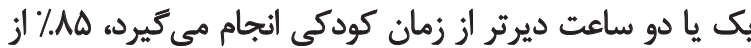

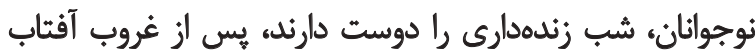

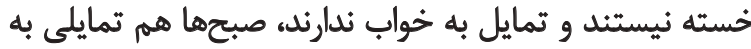

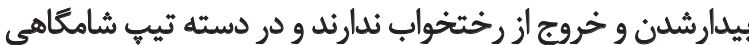

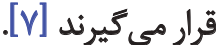

نوجوانى مرحله انتقالى ههمى است كه با تغييرات شديد

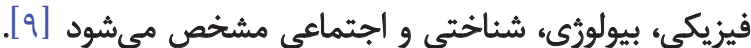

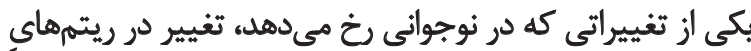

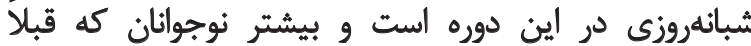

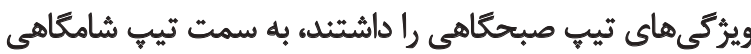

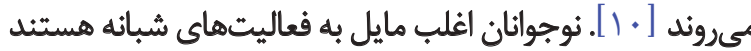

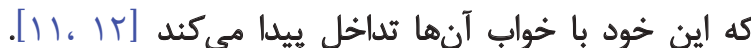

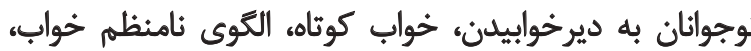

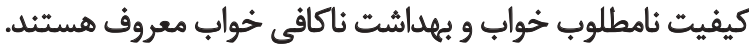

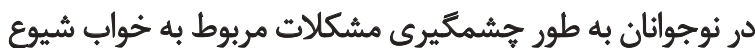

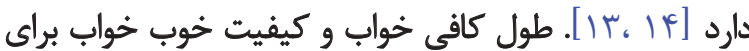

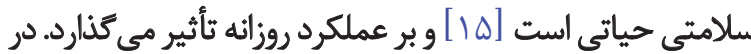

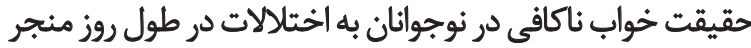

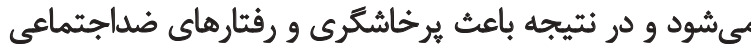

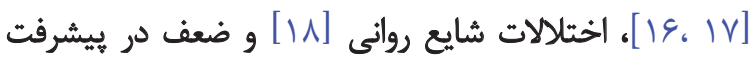
تحصيلى مىشود [9 19].

از جنبههاى اساسى عملكرد انطباقى انسان، توانايى كثترل

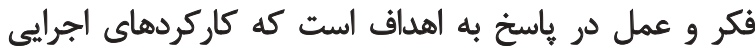

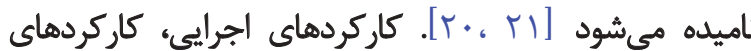

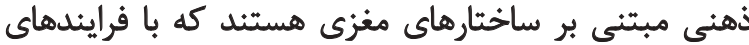

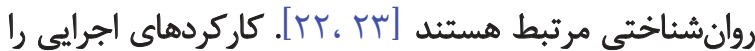

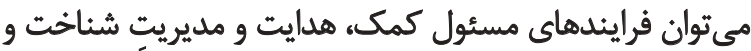




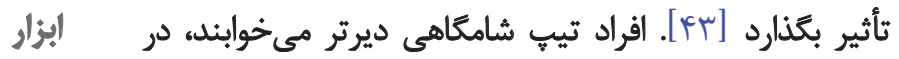

\section{يرسش نامه تيبزمانى صبحكَاهى شامكَاهى}

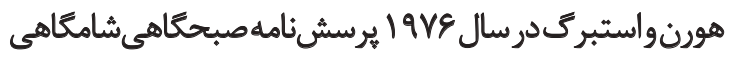

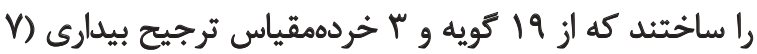

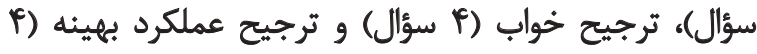

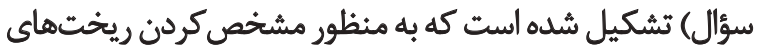

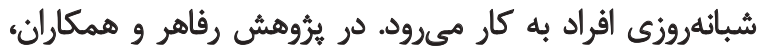

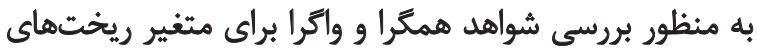

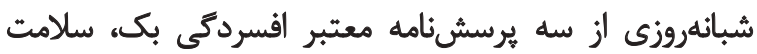

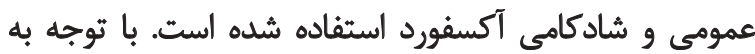

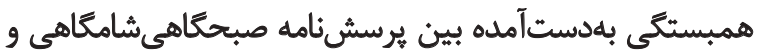

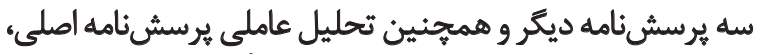

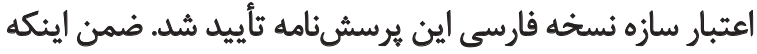

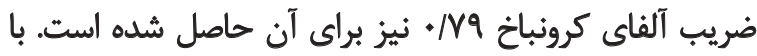

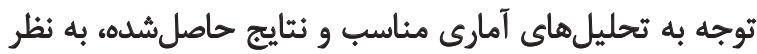

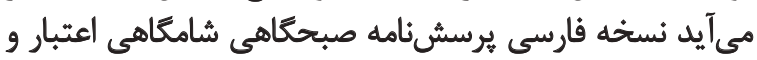

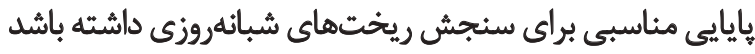

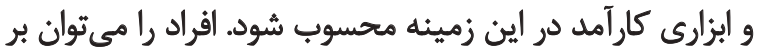

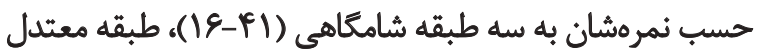

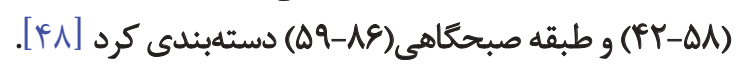

\section{يرسش نامه مقياس خطريذيرى نوجوانان ايرانى}

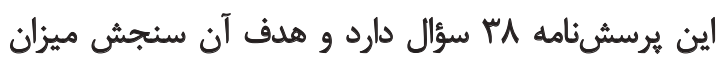

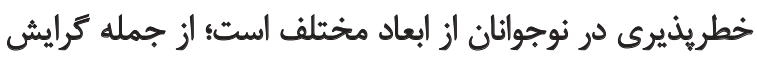

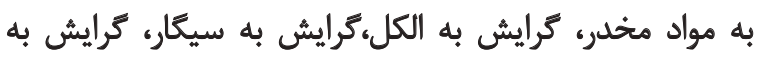

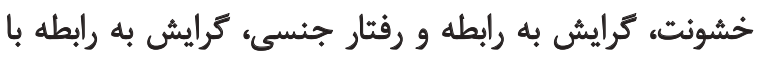

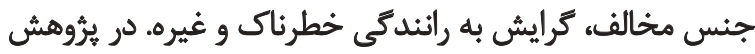

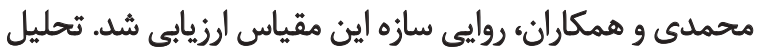

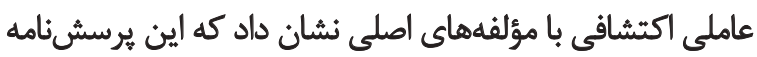

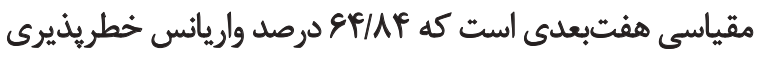

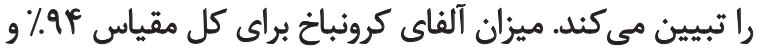

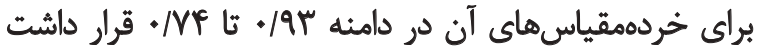

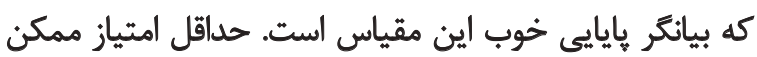

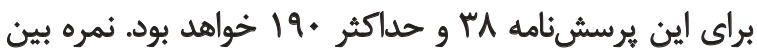

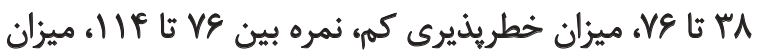

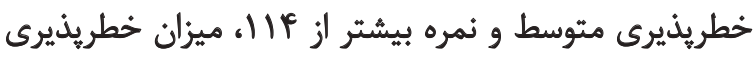

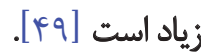

\section{هرسشنامه انغيزش ييشرفت تحصيلى}

يرسشنامه انكّيزش يُشرفت تحصيلى عبدخدايى در سال

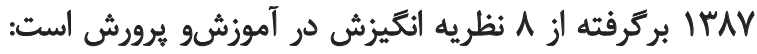

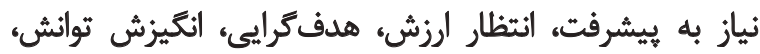

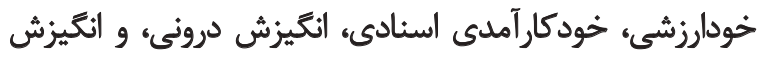

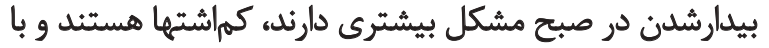

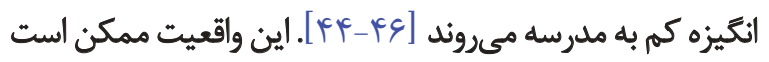
عملكرد تحصيلى تيب شامعاهى راتحث تأثير قرار دهد [ifV]

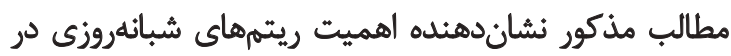

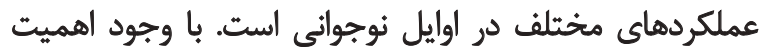

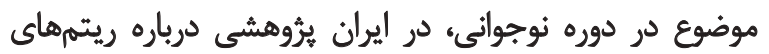

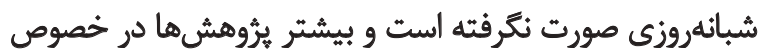

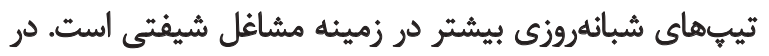

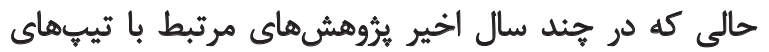

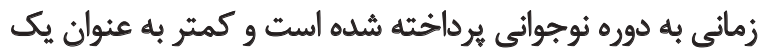

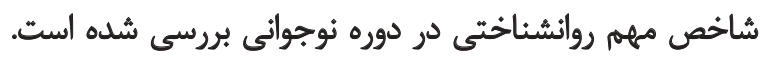

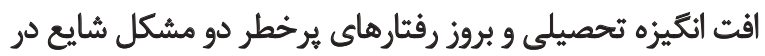

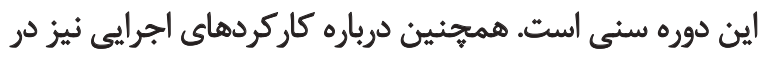

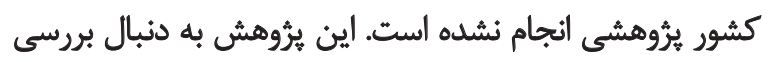

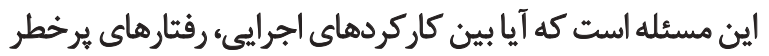

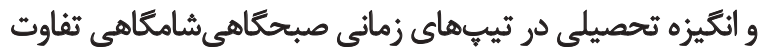

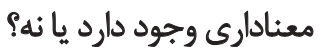

ووش

جامعه ونمونه

طرح يثرهش حاضر از نوع مقايسهاي بود و جامعه آمارى آن را

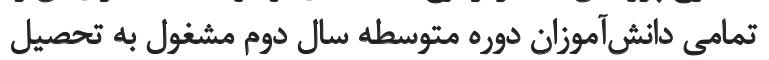

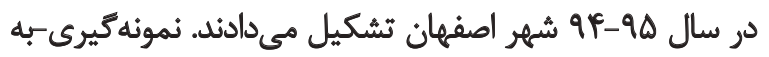

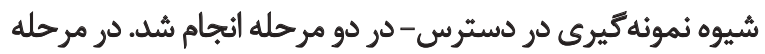

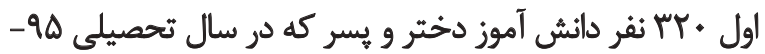

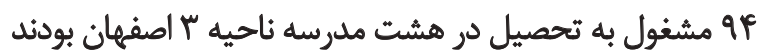

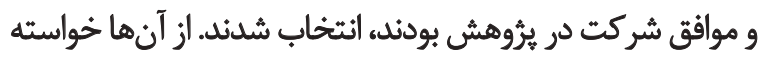

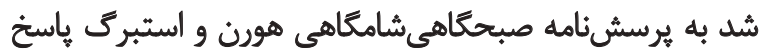

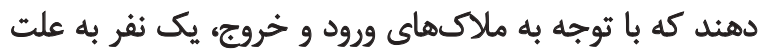

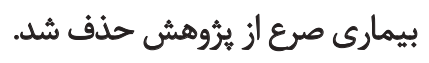

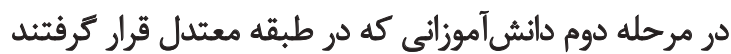
(نمره بين

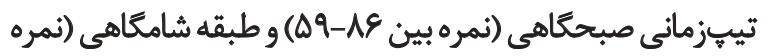

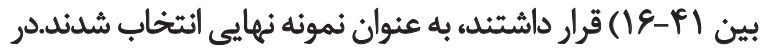

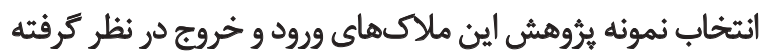

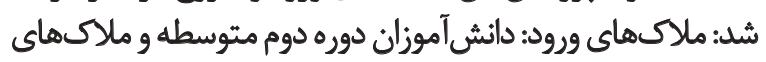

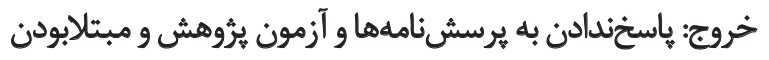

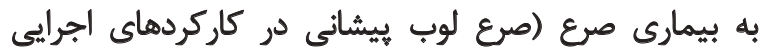

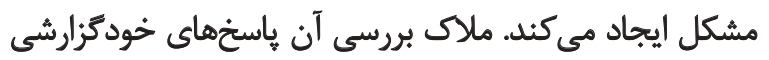
دانش آموزان بود). 
ساعد هاشمى) از كسانى كه مايل به شركت در بثروهش بودنده

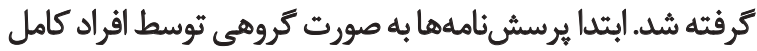

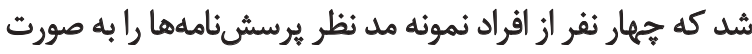

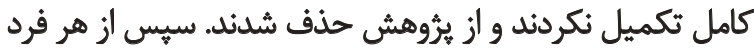

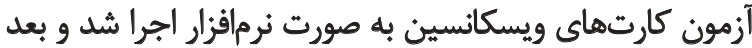

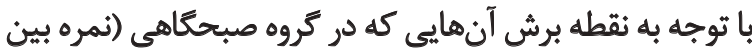

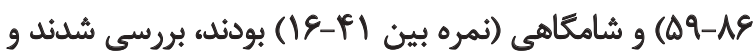

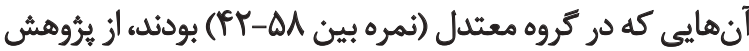

حذف شدند.

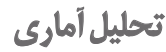

با بتفاده از نكارش سب نرم افزار SPSS در دو قسمت آمارههاي

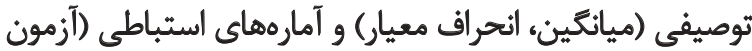

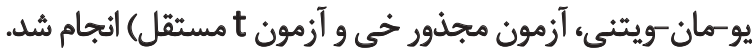

يأقتهها

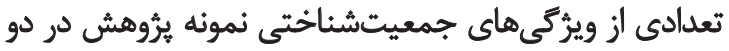

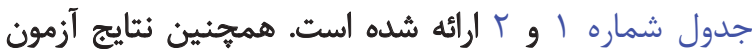

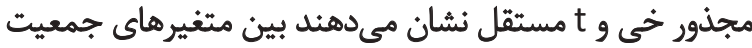

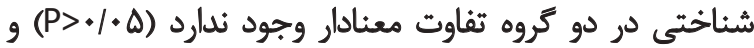

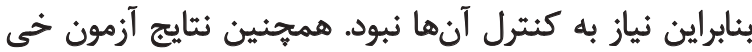

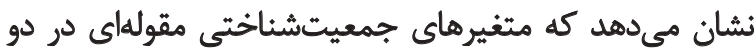

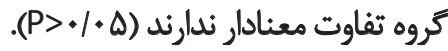

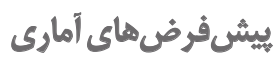

بيشفرض عادىبودن توزيع نمرات با استفاده از آزمون

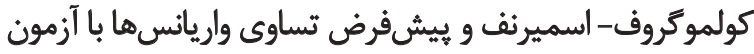

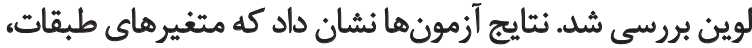
كوششها ، كوشش الكوى اول، مواد مخدر، الكل، سيكار و رابطه

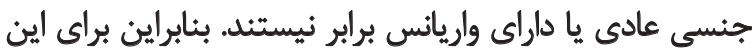

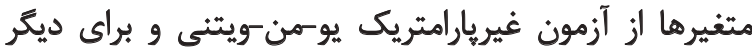

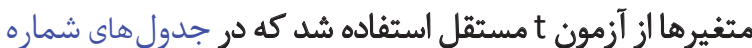

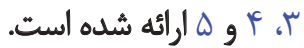

نتايج جدول شماره ؟ نشان مي دهد كه آماره Z در متغيرهاى

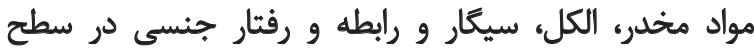

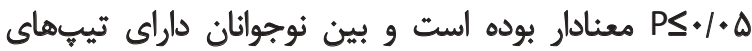

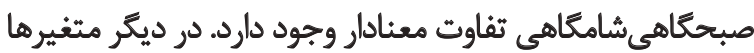

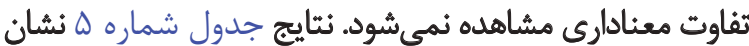

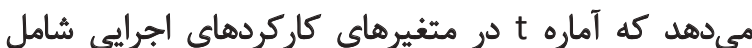

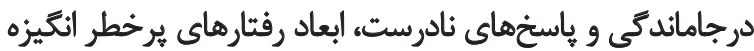

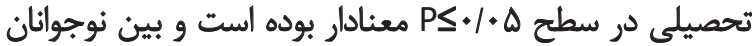

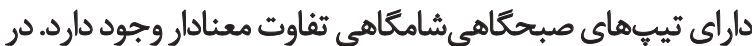

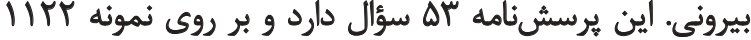

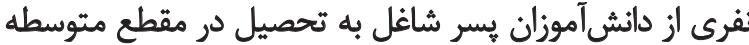

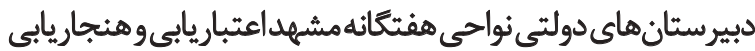

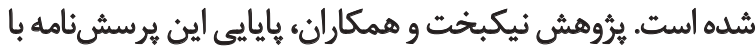

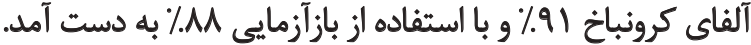

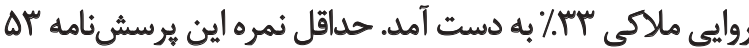

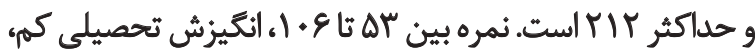

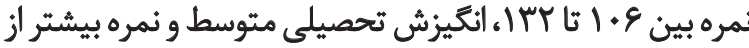

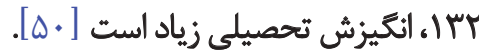

\section{آزمون دستدبندى كارتهاى ويسكانسين}

بركى و همكارانش در سال 9 ه ا نسخه اوليه آزمون دستهبندى

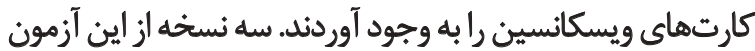

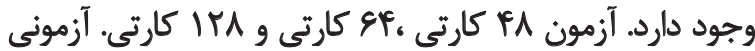

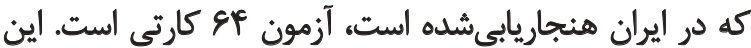

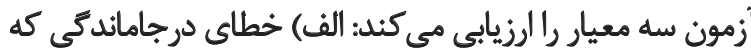

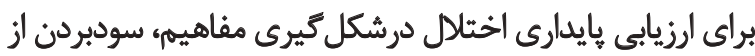

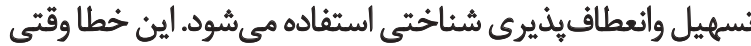

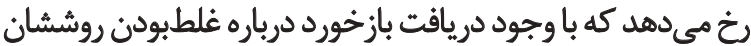

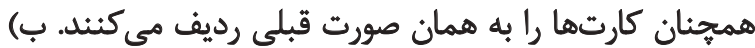

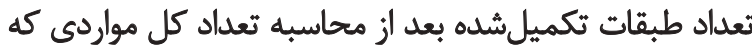

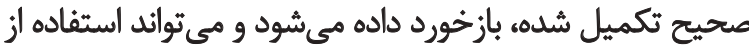

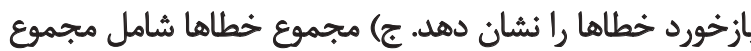

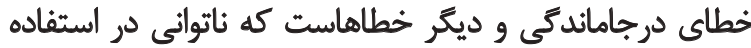

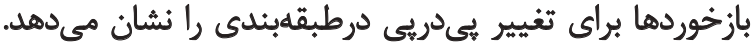

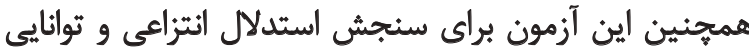

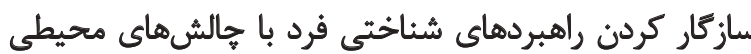

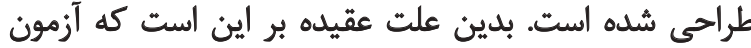

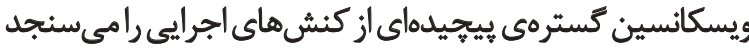

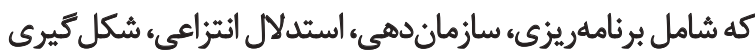

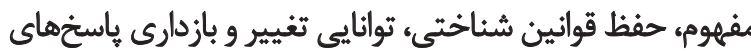

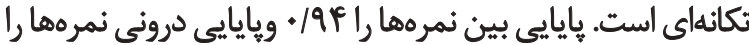

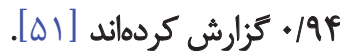

شيوهاجرا

ابتدا از دانشكاه محل تحصيل، مجوز لازم براى اداره

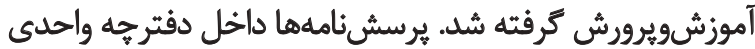

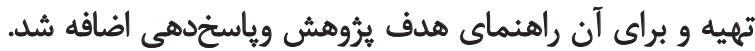

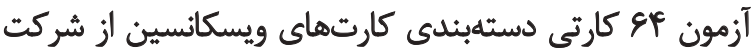

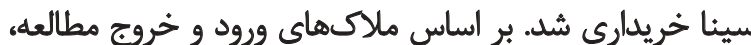

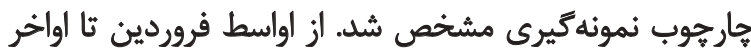

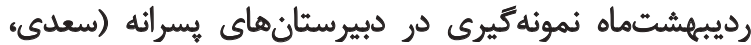

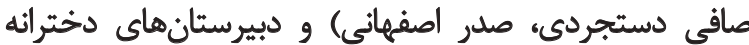

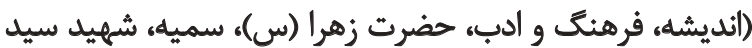




\begin{tabular}{|c|c|c|c|c|c|}
\hline \multicolumn{2}{|c|}{ آزمون جُى } & \multirow{2}{*}{ فراوانى (دراهى } & \multirow{2}{*}{ فببكَاهى } & \multirow[b]{2}{*}{ متغير } & \multirow{2}{*}{ كروه } \\
\hline معنادارى & ورجه آزادى & & & & \\
\hline \multirow{2}{*}{ - Mef } & \multirow{2}{*}{1} & $M(Y \cdot / V)$ & $r e(m / a)$ & ن & \multirow{2}{*}{ جنس } \\
\hline & & $1 /(1) / 9)$ & $r \cdot(Y N Y)$ & 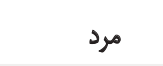 & \\
\hline \multirow{3}{*}{.$/ 19$} & \multirow{3}{*}{$r$} & $\Delta(\varphi / V)$ & $W(1 E / 9)$ & تجوبي & \multirow{3}{*}{ رشته تحصيلي } \\
\hline & & $\mid f(\mid r / Y)$ & $M(1 E / 9)$ & رياضى & \\
\hline & & $r(19 / 1)$ & $r \cdot($ YND $)$ & أنسائنى & \\
\hline \multirow{4}{*}{. Mrqq } & \multirow{4}{*}{$r$} & • & $\Delta(f / V)$ & بيسواد & \multirow{4}{*}{ تحصيلات هلحر } \\
\hline & & $T Y\left(T \Delta / T^{\prime}\right)$ & $F \backslash(Y N E)$ & دييلم & \\
\hline & & $1 \cdot(9 / 4)$ & $\mid r(\mid 1 / T)$ & ليسائس & \\
\hline & & $r(T / A)$ & $A(V / \Delta)$ & دكترى & \\
\hline \multirow{4}{*}{.$/ \Delta M$} & \multirow{4}{*}{$r$} & $1(\cdot / 99)$ & $P(T / M)$ & بيسواد & \multirow{4}{*}{ تحصيلات مادر } \\
\hline & & rI(rq) & 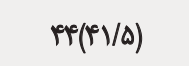 & دييلم & \\
\hline & & $V(\& / \&)$ & $\mid F(\mid r / T)$ & ليسائس & \\
\hline & & $1(\cdot / 94)$ & $r(r / V)$ & دكترى & \\
\hline \multirow{3}{*}{$\cdot 10 V^{2}$} & \multirow{3}{*}{ r } & $M(I Y / A)$ & $r g(m \Psi / q)$ & 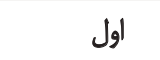 & \multirow{3}{*}{ ترتيب تولد } \\
\hline & & $r(T / \Lambda)$ & $r(Y / A)$ & دوק & \\
\hline & & $18(10)$ & rA(TE/P) & ل & \\
\hline
\end{tabular}

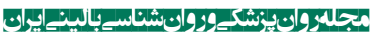

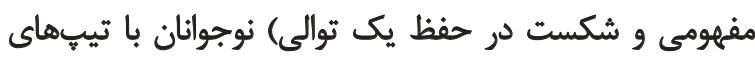

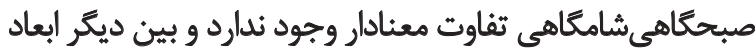

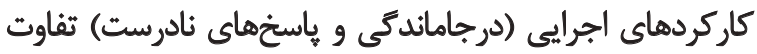

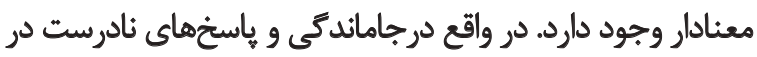
نوجوانان ثيبهاي شامكاهى بيشتر است.

در خصوص تيبهاى زمانى و متغير كاركردهاي اجرايى تحقيقات اندكى صورت كرفته است. هاهن و و همكاني

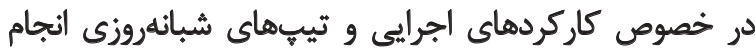

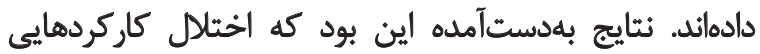

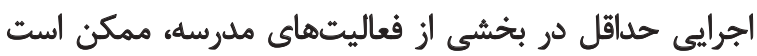

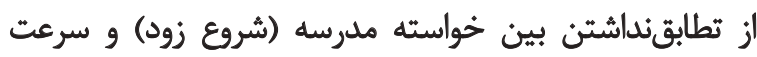
تغييرات ريتم شبانهروزى (تغيير به سمت شامعاهى) در طول ولن سرع
ديكر متغيرها تفاوت معنادارى مشاهده نمىشود.

بحث

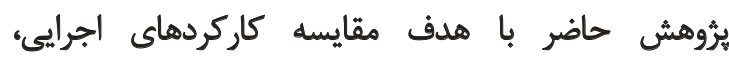

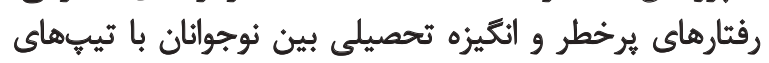

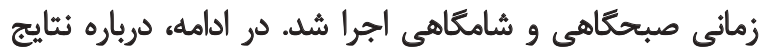

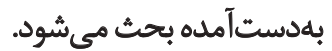

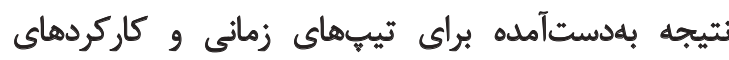

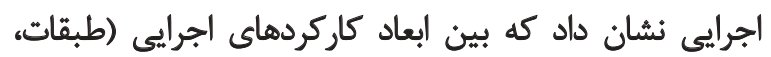

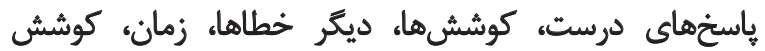

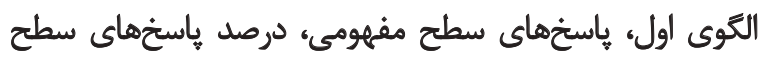

جدول r. فراواني و درصد متغيرهاي جمعيثشئاختي فاصلهاي به تفكيك دو كروه

\begin{tabular}{|c|c|c|c|c|}
\hline آزمون t مستقل & + & شامكاهى & صبحكَّاهى & \\
\hline معنادارى & & ميانكيني(انحر اف معيار) & هيانكين (انحراف معيار) & مثغير \\
\hline . MAF & $+/ V^{2}$ & $19(1)$ & $\operatorname{IV}(1)$ & معدل \\
\hline$\cdot / r+1$ &.$/ M$ & $f(\cdot)$ & $f(\cdot)$ & تعداد اعضاى خانواده \\
\hline
\end{tabular}

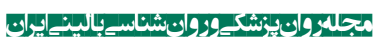




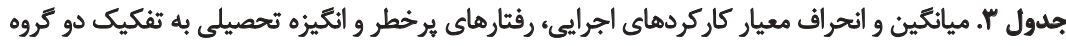

\begin{tabular}{|c|c|c|c|c|c|c|c|}
\hline \multicolumn{3}{|c|}{ شاهمكاهى } & \multicolumn{3}{|c|}{ صبحكاهى } & \multirow[b]{2}{*}{ متغير } & \multirow[t]{2}{*}{ كرو } \\
\hline ميانكين (انحراف معيار) & حداكثر & حداقل & ميانكين (انحراف معيار) & حداكثر & حداقل & & \\
\hline$r(\nu)$ & 8 & . & $r(1)$ & $\&$ & • & طبقات & \\
\hline$q\left({ }^{\infty}\right)$ & M & - & $A\left(P^{\oplus}\right)$ & M & 1 & درجاماندكى & \\
\hline$\Pi r(\varphi)$ & Pr & r. & $r+(\varepsilon)$ & pr & 10 & ياسخ درست & \\
\hline$r \varepsilon(A)$ & p. & 8 & $r q(V)$ & Pa & in & ياسخنادرست & \\
\hline$\Delta \wedge(Y)$ & \&. & Pa & $\Delta q(\mu)$ & 81 & rq & كل كوثش ها & \\
\hline $19(\Delta)$ & r. & 8 & $r\left(r^{*}\right)$ & r+ & 1. & ساير خطاها & كاركردهاي اجرايي \\
\hline$r+r\left(M^{e}\right)$ & $\Delta+\Lambda$ & in & MIV(AT) & gr. & Mr & زمان & \\
\hline$I V(I r)$ & q. & 8 & $M r(M)$ & q. & 8 & كوشش التوى اول & \\
\hline$r(\tau)$ & 8 & . & $Y(Y)$ & $\varepsilon$ & - & ثاسخخهاي سطحمفهومي & \\
\hline$\Delta 1(19 q)$ & $1 \ldots$ & . & Pr(feg) & 1.0 & . & درصدياسخ ها سطح مفهوهي & \\
\hline$r(\cdot)$ & . & . & $r(\cdot)$ & - & . & شكست درحفظ يكتوالى & \\
\hline$M(M Y)$ & IVA & PV & $\operatorname{er}(I V)$ & ११ & & رفتارهاى يرخطر & \\
\hline$M(\Delta)$ & ו & $\wedge$ & $q(Y)$ & 18 & & مواد مخدر & \\
\hline $\operatorname{If}(\lambda)$ & f. & 8 & $q\left(P^{e}\right)$ & r & & الكل & \\
\hline$V(\%)$ & rr & $\Delta$ & $g(r)$ & 19 & & سيكار & \\
\hline $\mid r(\varphi)$ & ta & 8 & $q(\Psi)$ & m & & خشونت & رفتمارهاى \\
\hline $9(\%)$ & r. & p & $q(r)$ & iv & & رفتار جنسى & \\
\hline$\| r(r)$ & r. & 8 & $q\left(F^{F}\right)$ & $r+$ & & دوستى با جنس مخالف & \\
\hline$r \cdot(\Delta)$ & r. & $\wedge$ & $1 f(9)$ & r. & & رانتدكى خطرناك & \\
\hline $1 F 8(19)$ & 19 & qf & $\mid \varepsilon q(r \cdot)$ & $r+r$ & & انكيزه تحصيلى & \\
\hline
\end{tabular}

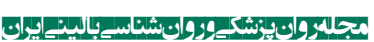

افراد تيب شامكاهى در مقايسه با افراد تيب صبحكاهى با ثأخير

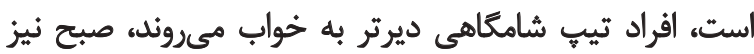

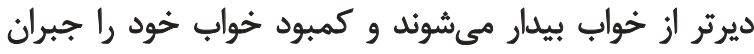

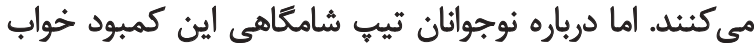

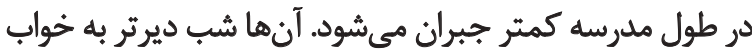

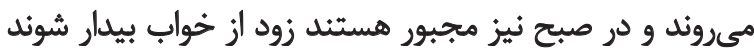

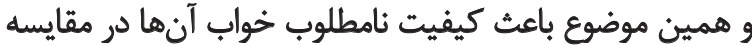

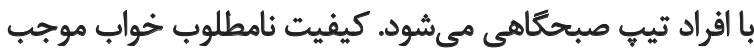

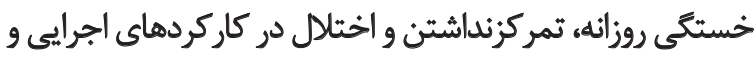

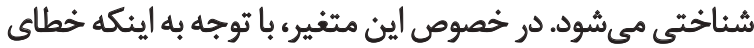

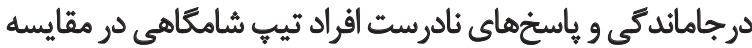

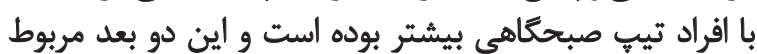

انتقال به نوجوانى باشد. بنابراين كاركردهايى اجرايى ممكن

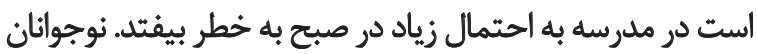

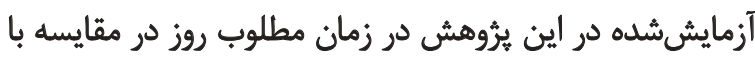

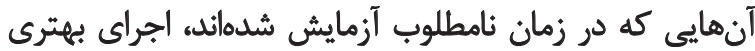

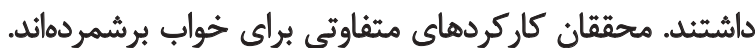

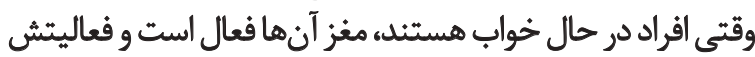

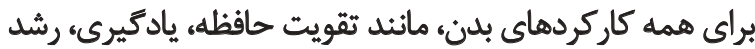

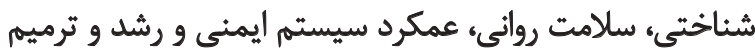

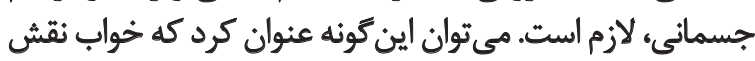
مهمى در فعاليتهاى روزانه افراد دارد. با توجه به اين كه ريتمهاى خواببيدارى و فعاليت|ستراحت 
جدول F. نتايج آزمون يومن-ويتنى در مقايسه متغيرهاى بُؤهش بين دو كروه

\begin{tabular}{|c|c|c|c|c|}
\hline \multicolumn{5}{|c|}{ أزمون يو-من-ويتنى } \\
\hline معنادارى & $z$ & شامكاهى & صبحتَاهى & مثغير \\
\hline ./NTr & V/AP & $\Delta q / T A$ & $\Delta . /$. & تعلداد طبقات \\
\hline . TYA & $1 / .9$ & $\mathrm{eq} / \mathrm{TT}$ & $\Delta 81 . \mu$ & كوشش الكوى اول \\
\hline $.1 .0 r$ & $T / 10$ & ge/va & Peles & هواد هخلر \\
\hline $.1 . .1$ & $r / P r$ & selt. & PD/FA & الكل \\
\hline 1.4 & $r / . \Delta$ & $\varepsilon \cdot \pi$ & $p q /{ }^{\prime} \wedge$ & سيكار \\
\hline $.1 . .1$ & $r / 4 \&$ & gs/r. & $r \Delta / V^{f}$ & رابطه و رفتار جنسى \\
\hline
\end{tabular}

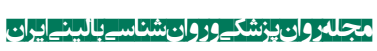

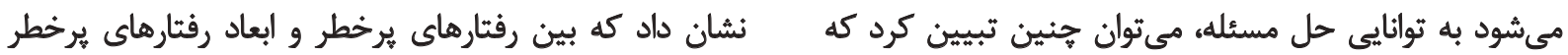

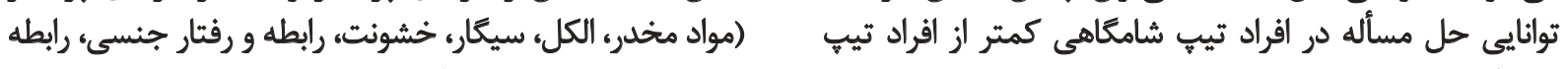

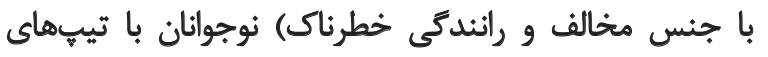
صبحكاهى است.

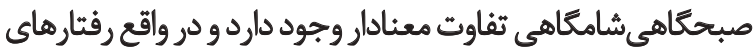

نتيجه بهدست آمده براى تيبهاى زمانى و رفتارهاى برخطر

جدول ه. نتايج آزمون t مستقل در مقايسه متغيرهاى برؤوهش بين دو كروه

\begin{tabular}{|c|c|c|c|c|c|c|c|}
\hline \multicolumn{8}{|c|}{ أزمون t } \\
\hline \multicolumn{2}{|c|}{ سطح اطمينان 90 درصد } & \multirow[b]{2}{*}{ معنادارى } & \multirow{2}{*}{ آزاديه } & \multirow{2}{*}{$\mathrm{t}$} & \multirow{2}{*}{ ميانكين (انحراف معيار) } & \multirow{2}{*}{ 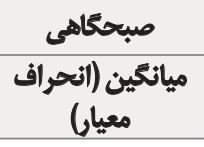 } & \multirow{2}{*}{ متغير } \\
\hline برالان & كران & & & & & & \\
\hline$r / F A$ &.$/ I V e$ & /.M & $1 .+5$ & $r / 19$ & $g / q V(F / 19)$ & $N A \cdot(F / N 9)$ & درجاماندىى \\
\hline . MTS & -r/ar & $\% \cdot 1 \Delta$ & 1.8 & $-1 / N$ & $T r / r \cdot(g / T \Delta)$ & $r \cdot 1 \cdot .(g / 1))$ & مياسخهاى درست \\
\hline$\Delta / q u$ & $\% v$ &.$/ p q$ & $1 . f^{2}$ & V/MM & re/V.(NTI) & ra/sq $(Y / \cdot)$. & هاسخ هاى نادرست \\
\hline$r / \Lambda$. & / /AY & / RN & 1.4 & $\cdot|A V|$ & $\Delta N V A(Y / T V)$ & $\Delta q / r q(r / q))$ & كل كوششها \\
\hline r/Ps & ג &.$M E$ & $1 .+$ & $V / \Delta A$ & $19 / \Gamma V(\Delta / \mathscr{R T})$ & $r V / * 1(\varphi / m)$ & ساير خطاها \\
\hline$\varphi g / V$ & $-18 / T$ &.$/ \pi T$ & $1 . f$ &.$/ 9 \Delta F$ & $r \cdot r / r \cdot(M+/ 8 \Lambda)$ & TIV/Q.(AT/Tg) & زمان \\
\hline ./FrA & $-1 / A r$ &.$/ \pi r$ & 1.4 & $-1 / \pi$ & $r / T \Delta(T / Q 9)$ & $T / \Delta F(T / M A)$ & خاسخ هاى سطح مفهومى \\
\hline $9 / \Delta$. & -TNTA &.$/ M r V$ & 1.8 &.$- / 44$ & $\Delta \backslash / q \Delta(F q / \Psi \varepsilon)$ & 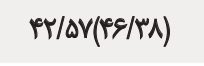 & درصد ياسخخ هاى سطح مفهومى \\
\hline 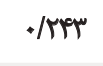 & -1101 & IFVG & 1.8 &.$/ 419$ & $\cdot / r \cdot(\cdot / \Delta \mid \Delta)$ & $\cdot / M F Y(\cdot / F q \Lambda)$ & شكست در حفظ يك توالى \\
\hline$-|V /|$ & $-r T / q$ & $\%$ & 1.5 & $-8 / M$ & MWYT(TYT/TA) & $g T / N O(I V / T V)$ & رفتار هاي برخطر \\
\hline 1.89 & $-T / 9 \Delta$ & .1 .1 & 1.8 & $-1 / M$ & $\mid w / I V(f / g))$ & Q/PeA(r/Ar) & خشونت خ \\
\hline$-1 / r \Delta$ & $-r / 1 r$ & $\%$ & 1.8 & $-r / T \Delta$ & $Q / r(F / W)$ & $g / N r(r / l \cdot)$ & رابطه با جنس مخالف \\
\hline$-r / N$ & $-N P 1$ & $.1 .+1$ & $1 .+5$ & $-\phi / \omega$ & $r \cdot R \Delta(\Delta / Q V)$ & $\mid P / R Y(\& / 1 A)$ & رائندى خطرناك \\
\hline rN.. & $\mid Y / \cdot 9$ & 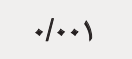 & 1.5 & r/ar & $\mid \& \& / 1(19 / 94)$ & $\operatorname{lsg} / r(r \cdot M r)$ & انتيزه تحصيلى \\
\hline
\end{tabular}

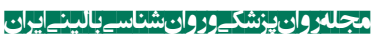


تحصيلى افراد تأثير بكذارد. بسيارى از برنامههاى آموزشى رو درٍ

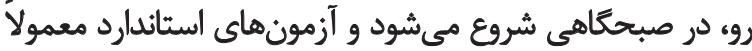

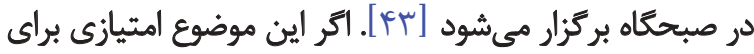

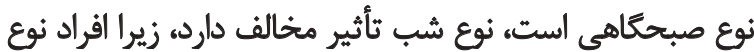

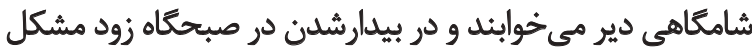

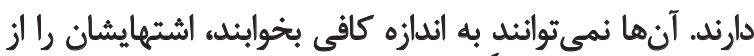

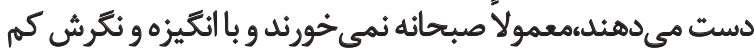

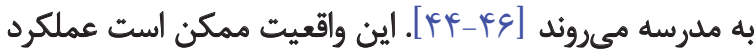

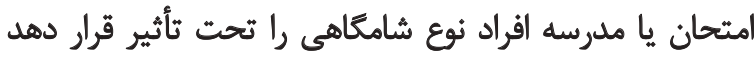

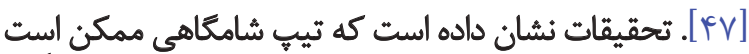

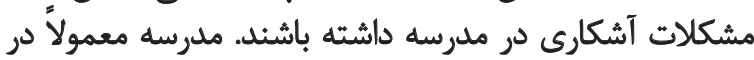

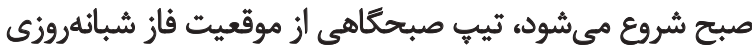

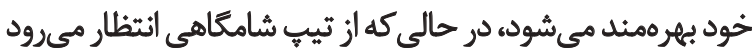

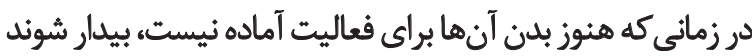

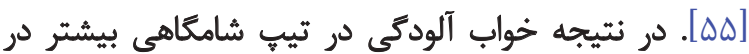

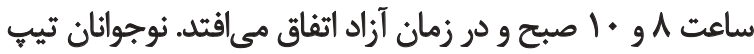

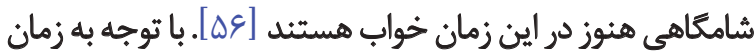

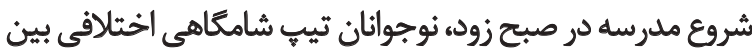

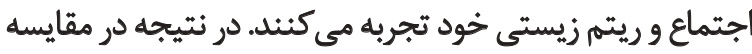

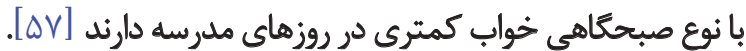

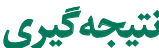

خواب ناكافى باعث كاهش توجه و افزايش مشكلات مربوط به

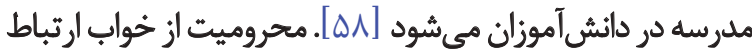

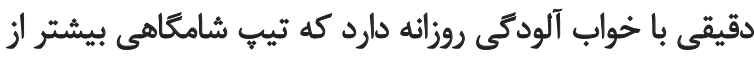

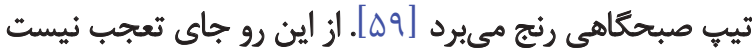

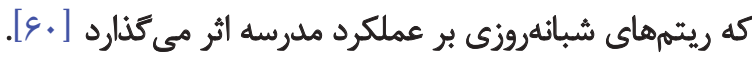

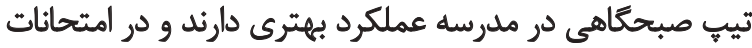

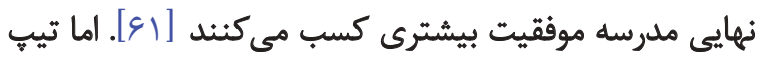

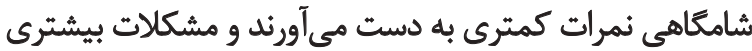

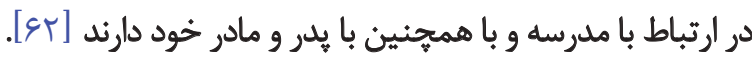

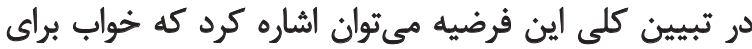

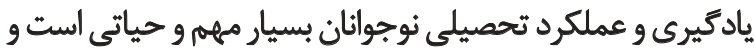

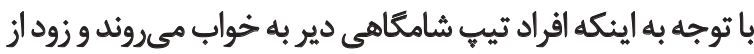

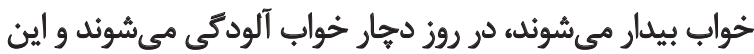

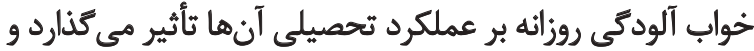

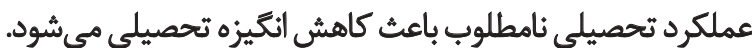

يكى از مهمترين محدوديتهاى اين ثروهش كمبود منابع

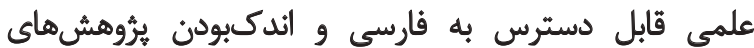

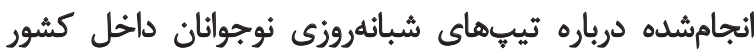

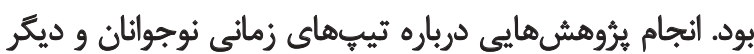

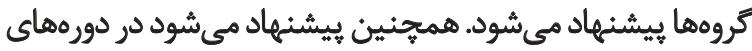

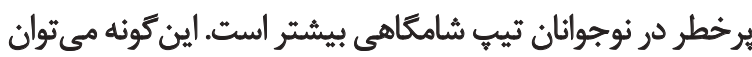

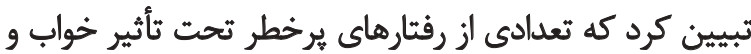

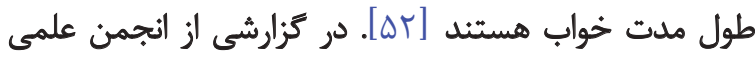

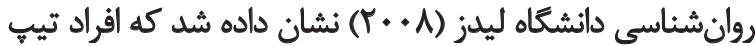

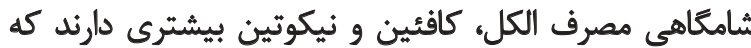

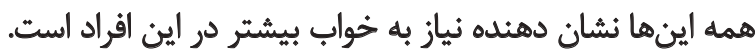

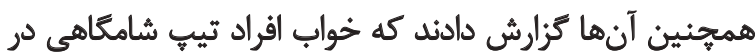

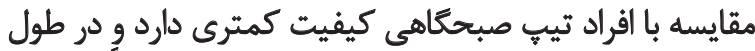

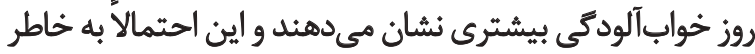

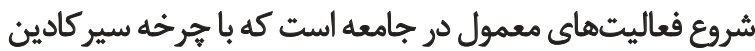

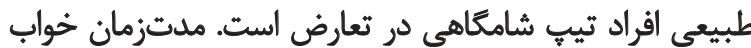

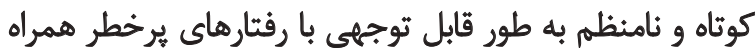

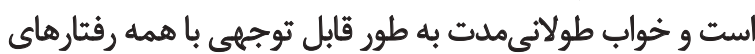

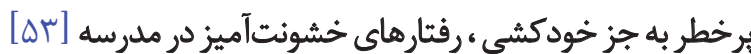

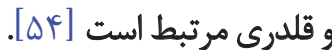

تحقيقات هايوا و همكاران نشان داد كه استفاده بيش از حد از

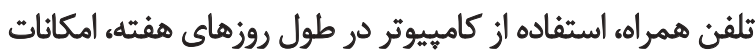

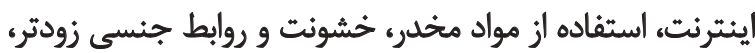

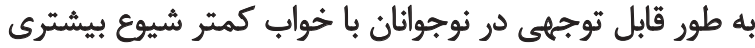

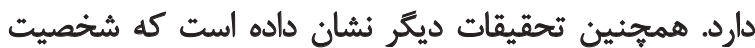

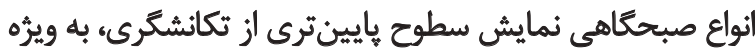

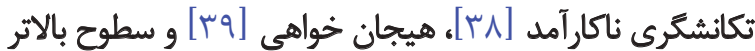

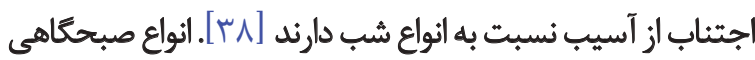

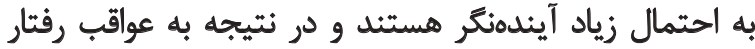

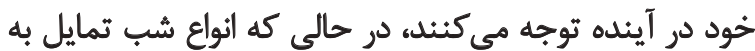

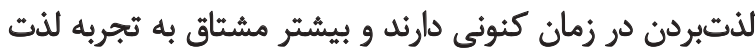

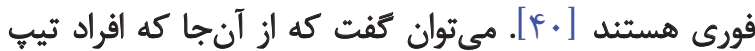

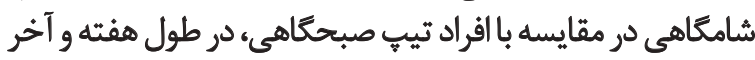

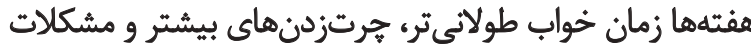

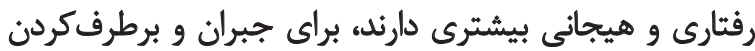

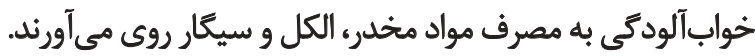
نتيجه بهدستآمده براي تيبهاي زمانى و انتيزه تحصيلى تئى تئي

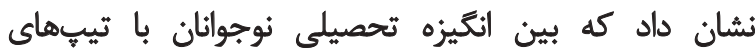

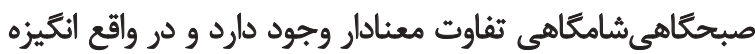

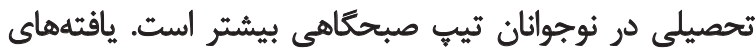

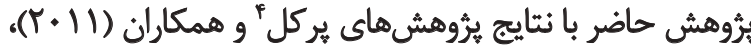

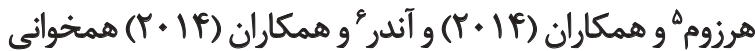

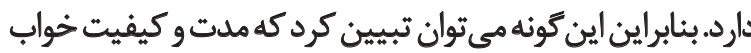

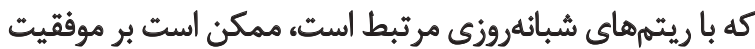


آموزشى، تيبهاى شبانهروزى افراد در نظر ترفته شود و افراد

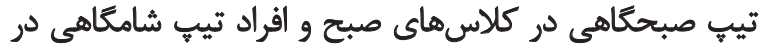

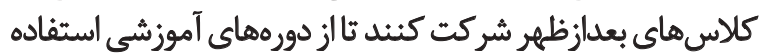

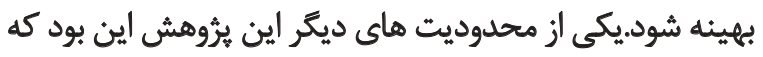

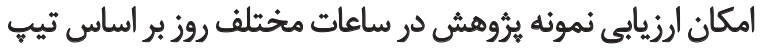

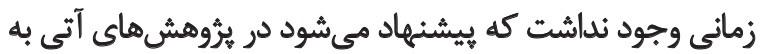
اين زمينه توجه شود.

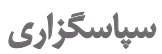

از استادان و همجنين تمامى دانشجويان شركت كنينده در

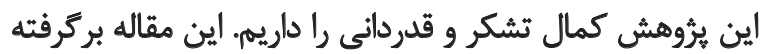

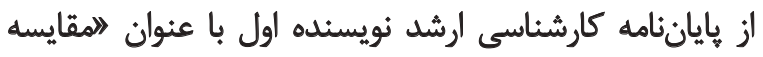

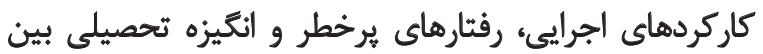

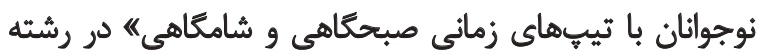

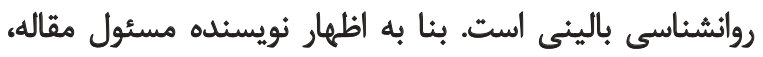
تعارض منافع و حمايت مالى از بثروهش وجود نداشته است نواسن 


\section{References}

[1] Mecacci L, Righi S, Rocchetti G. Cognitive failures and circadian typology. Personality and Individual Differences. 2004; 37(1):107-13. doi: 10.1016/j.paid.2003.08.004

[2] Caci H, Robert P, Boyer P. Novelty seekers and impulsive subjects are low in morningness. European Psychiatry. 2004; 19(2):79-84. doi: 10.1016/j.eurpsy.2003.09.007

[3] Diaz-Morales, JF. (2007). Morning and evening type: Exploring their personality styles. Personality and Individual Differences. 43(4):769-778

[4] Ziaei M, Amiri S, Molavi H. [Relationship between score of circadian types and time of student reaction at morning and evening (Persian)]. Advances in Cognitive Science .2007; 9(2):47-53.

[5] Cavallera GM, Giudici S. Morningness and eveningness personality: A survey in literature from 1995 up till 2006. Personality and Individual Differences. 2008; 44(1):3-21. doi: 10.1016/j.paid.2007.07.009

[6] Tonetti L, Fabbri M, Natale V. Relationship between circadian typology and big fire personality domains. Chronobiology International. 2009; 26(2):337-47. doi: $10.1080 / 07420520902750995$

[7] Randler C, Frech D. Young people's time-of-day preferences affect their school performance. Journal of Youth Studies. 2009;12(6):653-67. doi:10.1080/13676260902902697

[8] Díaz-Morales JF, Pilar Sánchez-López M. Morningnesseveningness and anxiety among adults: A matter of sex/ gender? Personality and Individual Differences. 2008; 44(6):1391-401. doi: 10.1016/j.paid.2007.12.007

[9] Lalonde G, Henry M, Drouin-Germain A, Nolin P, Beauchamp $\mathrm{MH}$. Assessment of executive function in adolescence: A comparison of traditional and virtual reality tools. Journal of Neuroscience Methods. 2013; 219(1):76-82. doi: 10.1016/j.jneumeth.2013.07.005

[10] Goldstein D, Hahn CS, Hasher L, Wiprzycka UJ, Zelazo PD. Time of day, intellectual performance, and behavioral problems in Morning versus Evening type adolescents: Is there a synchrony effect? Personality and Individual Differences. 2007; 42(3):431-40. doi: 10.1016/j.paid.2006.07.008

[11] Arora T, Broglia E, Thomas GN, Taheri S. Associations between specific technologies and adolescent sleep quantity, sleep quality, and parasomnias. Sleep Medicine. 2014; 15(2):240-7. doi: 10.1016/j.sleep.2013.08.799

[12] Demirhan E, Randler C, Horzum MB. Is problematic mobile phone use explained by chronotype and personality? Chronobiology International. 2016; 33(7):821-31. doi: 10.3109/07420528.2016.1171232

[13] Diaz-Morales JF, Escribano C. [Consequences of adolescents evening preference on school achievement: A review (Spanish)]. Anales de Psicologia. 2014; 30(3):1096-1104.doi: 10.6018/analesps.30.3.167941

[14] Russo PM, Bruni O, Lucidi F, Ferri R, Violani C. Sleep habits and circadian preference in Italian children and adolescents. Journal of Sleep Research. 2007; 16(2):163-9. doi: 10.1111/j.1365-2869.2007.00584.x
[15] Jarrin DC, McGrath JJ, Drake CL. Beyond sleep duration: distinct sleep dimensions are associated with obesity in children and adolescents. International Journal of Obesity. 2013; 37(4):552-8. doi: 10.1038/ijo.2013.4

[16] Schlarb AA, Sopp R, Ambiel D, Grünwald J. Chronotype-related differences in childhood and adolescent aggression and antisocial behavior - A review of the literature. Chronobiology International. 2013; 31(1):1-16. doi: 10.3109/07420528.2013.829846

[17] Simor P, Zavecz Z, Pálosi V, Török C, Köteles F. The influence of sleep complaints on the association between chronotype and negative emotionality in young adults. Chronobiology International. 2014; 32(1):1-10. doi: 10.3109/07420528.2014.935786

[18] Haregu A, Gelaye B, Pensuksan WC, Lohsoonthorn V Lertmaharit S, Rattananupong T, et al. Circadian rhythm characteristics, poor sleep quality, daytime sleepiness and common psychiatric disorders among Thai college students. Asia-Pacific Psychiatry. 2014; 7(2):182-9. doi: 10.1111/appy.12127

[19] Tonetti L, Natale V, Randler C. Association between circadian preference and academic achievement: A systematic review and meta-analysis. Chronobiology International. 2015; 32(6):792-801. doi: 10.3109/07420528.2015.1049271

[20] Diamond A. Executive functions. Annual Review of Psychology. 2013; 64(1):135-68. doi: 10.1146/annurevpsych-113011-143750

[21] Zelazo PD, Anderson JE, Richler J, Wallner-Allen K, Beaumont JL, Weintraub S. II. NIH toolbox cognition battery $(\mathrm{cb})$ : measuring executive function and attention. Monographs of the Society for Research in Child Development. 2013; 78(4):16-33. doi: 10.1111/mono.12032

[22] Alizadeh H. [Neuro-cognitive executive functions related to developmental disorders (Persian)]. Advances in Cognitive Science. 2006; 8(4): 57-70.

[23] Loftiz W Christopher. An ecological validity study of executive function measures in children with and without attention deficit hyperactivity disorder [PhD dissertation]. Gainesville, Florida: University of Florida; 2004.

[24] Anderson T. Modes of interaction in distance education: Recent developments and research questions. In Moore M editor. Handbook of Distance Education. 2012; Abingdon: Routledge.

[25] Fuster JM. The pre frontal cortex. London: Academic Press; 2008.

[26] Gioia GA, Isquith PK, Guy SC, Kenworthy L. Behavior rating inventory of executive function. Child Neuropsychology. 2000; 6(3):235-38. doi: 10.1076/chin.6.3.235.3152

[27] Crone EA. Executive function in adolescence: Inferences from brain and behavior. Developmental Science. 2009; 12(6):825-30. doi: 10.1111/j.1467-7687.2009.00918.x

[28] Hahn C, Cowell JM, Wiprzycka UJ, Goldstein D, Ralph $\mathrm{M}$, Hasher L, et al. Circadian rhythms in executive function during the transition to adolescence: the effect of synchrony between chronotype and time of day. Developmental Science. 2012; 15(3):408-16. doi: 10.1111/j.14677687.2012.01137.x 
[29] Boyer TW. The development of risk-taking: A multi-perspective review. Developmental Review. 2006; 26(3):291345. doi: $10.1016 /$ j.dr.2006.05.002

[30] Del Vecchio T, Oleary KD. Effectiveness of anger treatments for specific anger problem: A meta-analytic review. Clinical Psychology Review. 2004; 24(1):15-34. doi: 10.1016/j.cpr.2003.09.006

[31] Doran N, Luczak SE, Bekman N, Koutsenok I, Brown SA. Adolescent substance use and aggression: A review. Criminal Justice and Behavior. 2012; 39(6):748-69. doi: $10.1177 / 0093854812437022$

[32] Gans J, Blyth D, Elster A, Gaveras LL. Americans adolescents: How healthy are they? (Vol. 1). Chicago, Illinois: American Medical Association; 1990.

[33] Irwin CE. Adolescence and risk taking: How are they related? In: Bell NJ, Bell RW. Adolescent Risk Taking. Thousand Oaks, California: Sage Publication; 1993.

[34] Marquez MPN, Galbon NT. Getting hotter, going wilder? Changes in sexual risk taking behavior. Paper presented at: The $7^{\text {th }}$ International Conference on Philippine Studies. 16-19 June 2004; Lieden, Netherlands.

[35] Jesser R. Risk behavior in adolescence: A Psychosocial framework for understanding and action. In: Rogers DE, Ginzberg E, editor. Adolescents At Risk: Medical And Social Perspectives. Boulder, Colorado: Westview Press; 1992.

[36] Lindberg LD, Boggess S, Williams S. Multiple threat: The co occurrence of teen health risk behaviors. Washington, D.C.: The Urban Institute; 2000.

[37] Bergman M, Scott I. Young adolescents well being and health risk behaviors: Gender and socioeconomic differences. Journal of Adolescence. 2001; 24(2):183-97. doi: 10.1006/jado.2001.0378

[38] Adan A, Natale V, Caci H, Part, G. Relationship between circadian typology and functional and dysfunctional impulsivity. Chronobiology International. 2010; 27(3):606-19. doi: $10.3109 / 07420521003663827$

[39] Tonetti L, Adan A, Caci H, De Pascalis V, febbri M, \& Natale V. Morningness-eveningness preference and sensation seeking. European Psychiatry. 2010; 25(2):111-15. doi: 10.1016/j.eurpsy.2009.09.007

[40] Stolarski M, Ledzinshka M, Matthews G. Morning is tomorrow is today: Relationships between chronotype and time perspective. Biological Rhythm Research. 2012; 44(2):181-96. doi: 10.1080/09291016.2012.656248

[41] Bentham S. Psychology and Education (Routledge Modular Psychology). Abingdon: Routledge; 2002.

[42] Sitpec Debrom, S. Motivation for Learning [R. Hassanzadeh, N. Amooie, Persian Trans]. Mashhad: Donyaye Pajooesh; 2001.

[43] Besoluk S. Morningness-eveningness perferences and university entyance examination scores of high school student. Personality and Individual Differences. 2001; 50(2):248-52. doi: 10.1016/j.paid.2010.09.038

[44] Jovanovski D, Bassili JN. The relationship between morningness-eveningness preference and online learn- ing. Biological Rhythm Research. 2007; 38(5):355-65. doi: 10.1080/09291010600950149

[45] Martin PY, Marrington S. Morningness-eveningness orientation, optimal time-of-day and attitude change: Evidence for the systematic processing of a persuasive communication. Personality and Individual Differences. 2005; 39(2):367-77. doi: 10.1016/j.paid.2005.01.021

[46] Randler C, Frech D. Correlation between morningness-eveningness and final school leaving exams. Biological Rhythm Research. 2006; 37(3):233-39. doi: 10.1080/09291010600645780

[47] Boschloo A, Ouwehand C, Dekker S, Lee N, De Groot $\mathrm{R}$, krabbendam $\mathrm{L}$, et al. The relation between breafart skipping and school performance in adolescents. Mind, Brain, and Education. 2012; 6(2):81-88. doi: 10.1111/j.1751228x.2012.01138.x

[48] Rahafar A, sadeghi jujilee M, Sadeghpour A, Mirzaei S. Surveying Psychometric Features of Persian Version of Morning-Eventide Questionnaire (Persian)]. Daneshvar Raftar. 2013; 2(8):109-122.

[49] Zadeh Mohammadi A, Ahmadabadi Z, Heidari M. [Construction and assessment of psychometric features of Iranian adolescents risk-taking scale (Persian)]. Iranian Journal of Psychiatry \& Clinical Psychology . 2011; 17(3):218-25.

[50] Nikbakht A, Abdekhodaee M, Hasanabadi H. [Effectiveness of reality therapy group counseling program on academic motivation and procrastination (Persian)]. Research in Clinical Psychology and Counselings. 2014; 3(2):81-94. doi: 10.22067/ijap.v3i2.15434

[51] Shahgholian M, Azadfalah P, Ashtiani A, Khodadadi M. [Design of the Wisconsin Card Sorting Test (WCST) computerized version: theoretical fundamental, developing and psychometrics characteristics (Persian)]. Studies of Clinical Psychology. 2011; 1(4):110-133.

[52] Padez C, Mourao I, Moreira P, Rosado V. Long sleep duration and childhood overweight/obesity and body fat. American Journal of Human Biology. 2009; 21(3):371-6. doi: 10.1002/ajhb.20884

[53] Hildenbrand AK, Daly BP, Nicholls E, Brooks-Holliday $\mathrm{S}$, Kloss JD. Increased risk for school violence-related behaviors among adolescents with insufficient sleep. Journal of School Health. 2013; 83(6):408-14. doi: 10.1111/ josh.12044

[54] Tochigi M, Nishida A, Shimodera S, Oshima N, Inoue K, Okazaki Y, et al. Irregular bedtime and nocturnal cellular phone usage as risk factors for being involved in bullying: A cross-sectional survey of Japanese adolescents. PLoS ONE. 2012; 7(9):e45736. doi: 10.1371/journal.pone.0045736

[55] Dahl RE, Lewin DS. Pathways to adolescent health sleep regulation and behavior. Journal of Adolescent Health. 2002; 31(6):175-84. doi: 10.1016/s1054-139x(02)00506-2

[56] Andrade MMM, Beneditosilva AA, Domenice S, Arnhold, IJP, Mennabarreto L. Sleep characteristics of adolescents: A longitudinal study. Journal of Adolescent Health. 1993; 14(5):401-6. doi: 10.1016/s1054-139x(08)80016-x

[57] Tzischinsky O, Shochat T. Eveningness, sleep patterns, daytime functioning, and quality of life in Israeli adoles- 
cents. Chronobiology International. 2011; 28(4):338-43. doi: 10.3109/07420528.2011.560698

[58] Fallone G, Acebo C, Seifer R, Carskadon MA. Experimental Restriction of Sleep Opportunity in Children: Effects on Teacher Ratings. Sleep. 2005; 28(12):1561-7. doi: 10.1093/sleep/28.12.1561

[59] Russo PM, Bruni O, Lucidi F, Ferri R, Violani C. Sleep habits and circadian preference in Italian children and adolescents. Journal of Sleep Research. 2007; 16(2):163-9. doi: 10.1111/j.1365-2869.2007.00584.x

[60] Giannotti F, Cortesi F, Sebastiani T, Ottaviano S. Circadian preference, sleep and daytime behaviour in adolescence. Journal of Sleep Research. 2002; 11(3):191-9. doi: 10.1046/j.1365-2869.2002.00302.x

[61] Randler C, Frech D. Correlation between morningness - eveningness and final school leaving exams. Biological Rhythm Research. 2006; 37(3):233-9. doi: 10.1080/09291010600645780

[62] Vollmer C, Schaal S, Hummel E, Randler C. Association among schoolrelated, parental and self-related problems and morningness-eveningness in adolescents. Stress and health. 2011; 27(5):413-9. doi: 10.1002/smi.1393 
